\title{
Estudo Demográfico do Câncer de Cólon e Reto no Estado de Sergipe
}

\section{Demographic Study of Colon and Rectum Cancer in the State of Sergipe}

\author{
JUVENALDAROCHATORRES NETO-TSBCP'; FÁBIO RAMOS TEIXEIRA ${ }^{3}$; ANACAROLINALISBOAPRUDENTE²; \\ CRISTIANE JESUS SILVINO ${ }^{3}$; JOFRANCIS SANTOS ARCIERE ${ }^{3}$; MÁRIO COSTA VIEIRAFILHO ${ }^{3}$ \\ ${ }^{1}$ Professor, Doutor e Chefe do Serviço de Colo-proctologia da Universidade Federal de Sergipe, ${ }^{2}$ Médica residente em \\ Colo-proctologia. Hospital Universitário, ${ }^{3}$ Acadêmico de medicina da Universidade Federal de Sergipe - BRASIL.
}

TORRES NETO JR; TEIXEIRA FR; PRUDENTE ACL; SILVINO CJ; ARCIERE JS; VIEIRA FILHO MC. Estudo Demográfico do Câncer de Cólon e Reto no Estado de Sergipe. Rev bras Coloproct, 2008;28(2): 215-222.

RESUMO: No Brasil, o câncer colorretal (CCR) foi a quinta causa de morte por câncer em homens e a quarta, em mulheres no período de 1995-1999. No entanto, há poucos registros sobre os aspectos epidemiológicos desta patologia em Sergipe, sendo assim foco de atenção desse estudo. Objetivo: Catalogar todos os casos de CCR diagnosticados no estado de Sergipe no período de 03 anos (2003-2005), traçando-se um estudo demográfico. Método: Os dados foram colhidos em laudos de todos os laboratórios de anatomia patológica do estado. Resultados: O CCR foi mais freqüente em mulheres $(\mathrm{n}=201 ; 56,62 \%)$ que em homens (n=154; $43,38 \%)$. A média de idade foi 60,27 anos, e 39 (10,99\%) tinham até 40 anos. Adenocarcinoma foi o tipo histológico mais encontrado, presente em $295(83,10 \%)$ pacientes. Em Sergipe, a incidência de CCR em 2003, 2004 e 2005 foi de 4,57; 4,72 e 5,29 por 100.000 homens e, 6,07; 5,57 e 6,98 por 100.000 mulheres respectivamente. Na capital, nos respectivos anos, esses números aumentaram para 10,25; 10,57 e 10,29 por 100.000 homens e 12,92; 13,16 e 15,07 por 100.000 mulheres. Conclusões: Os dados obtidos são semelhantes aos da literatura, mostrando um aumento nas incidências anuais da neoplasia.

Descritores: Câncer, cólon, reto, epidemiologia.

\section{INTRODUÇÃO}

O câncer colorretal (CCR) é responsável por grande parte dos óbitos na maioria das populações estudadas, inclusive no Brasil. Os dados do Sistema de Informações sobre Mortalidade do Ministério da Saúde indicaram, para o ano 2001, entre as causas de mortes definidas, a exceção das doenças do aparelho circulatório, as neoplasias como a primeira causa de óbito nas regiões Sudeste e Sul e a segunda, nas regiões Norte, Nordeste e Centro-Oeste, perdendo apenas para as causas externas ${ }^{(1)}$.

O Instituto Nacional do Câncer (INCA) informa que o câncer de cólon e reto entre 1995 e 1999 foi a quinta causa de morte por câncer nos homens, perdendo apenas para o acometimento do pulmão, estô- mago, próstata e esôfago. Entre as mulheres, se constituiu como a quarta causa de morte por câncer no país, perdendo apenas para mama, pulmão e estômago ${ }^{(2)}$.

O CCR apresenta uma ampla variação de freqüência em todo o mundo, e sua incidência tem-se aumentado nos países industrializados ${ }^{(3,4)}$. Os tumores malignos que acometem o cólon e o reto a cada ano somam cerca de 945 mil casos novos, sendo a quarta causa mais comum de câncer no mundo e a segunda em países desenvolvidos. No Brasil, o CCR é o quinto tumor mais incidente em homens e o quarto, em mulheres. Estima-se que o número de casos novos de câncer de cólon e reto para o Brasil em 2006 seja de 11.390 casos em homens e de $13.970 \mathrm{em}$ mulheres. Estes valores correspondem a um risco estimado de 12 casos novos a cada 100 mil homens e 15 para cada 100 mil mulheres ${ }^{(5)}$. 
Em Sergipe, estima-se para o ano 2006 que a taxa bruta de incidência por 100 mil seja de 3,40 para homens e 6,11 para mulheres. No entanto, esses números aumentam para 8,08 para homens e 11,60 para mulheres na capital ${ }^{(5)}$.

Em um estudo realizado por Neves et al, a mortalidade por câncer de cólon e reto é maior nas regiões Sul e Sudeste, ficando as regiões Norte e Nordeste com taxas de mortalidade cerca de duas vezes menores ${ }^{(6)}$, o que pode ser em decorrência da subnotificação e subdiagnóstico da doença.

No estado de Sergipe, pouco se sabe a respeito dos aspectos epidemiológicos desta patologia, o que pode estar levando a uma abordagem inadequada por parte das políticas de saúde pública, sendo indispensável à realização de um estudo epidemiológico no estado. Portanto, objetivamos, a partir dos arquivos de todos os laboratórios de Anatomia Patológica do Estado de Sergipe, realizar um estudo demográfico que traduza melhor a realidade da neoplasia colorretal.

\section{PACIENTES E MÉTODOS}

Trata-se de um estudo retrospectivo de todos os pacientes com diagnóstico de câncer de cólon e reto no estado de Sergipe em um período de 3 anos (2003 2005).

Os dados dos pacientes foram colhidos nos laudos de todos os laboratórios de anatomia patológica de Aracaju, cidade onde estão todos os laboratórios do estado. As informações foram colhidas de acordo com o protocolo para câncer de cólon e reto, tendo como principais variáveis: gênero, idade, procedência, raça, tipo histológico, grau de diferenciação e distribuição topográfica dos tumores. A partir desses dados, foi calculada a incidência anual.

Os dados foram pesquisados com a devida autorização dada pelos responsáveis das instituições citadas acima, através de documento que garante que o estudo manterá o sigilo sobre a identidade dos pacientes e que o mesmo não trará nenhum dano à instituição. Não foi necessário o uso do termo de consentimento livre e esclarecido assinado pelo paciente, pois o trabalho consistiu unicamente na coleta de dados contidos nos prontuários dos pacientes.

Após a coleta de dados, estes foram comparados para excluir possíveis pacientes que se encontraram descritos em mais de uma instituição, que serviriam de fonte de dados, em função da assistência multidisciplinar do câncer de cólon e reto.

A taxa de incidência anual foi calculada dividindo-se o número de casos novos (numerador) surgido no ano pela população residente (denominador) no ano considerado. A fonte para se obter a população residente foi o Instituto Brasileiro de Geografia e Estatística - IBGE ${ }^{(7)}$. A taxa de incidência anual também foi ajustada para o gênero, utilizando-se nesse caso o número de casos novos anuais e a população residente por gênero. A incidência foi relatada como o número de casos novos de câncer de cólon e reto por 100 mil habitantes.

Realizamos uma análise descritiva dos principais dados com planilhas elaboradas com o auxilio do Microsoft Excel ${ }^{\circledR} 2007$.

O estudo foi aprovado pelo Comitê de Ética em Pesquisa da Universidade Federal de Sergipe.

\section{RESULTADOS}

Foram encontrados 355 casos de câncer de cólon e reto confirmados pelos exames de anatomia patológica em um período de 3 anos, compreendidos entre janeiro de 2003 a dezembro de 2005 .

O CCR foi mais freqüente em mulheres $(n=201 ; 56,62 \%)$ que em homens $(n=154 ; 43,38 \%)$ (figura 1). A idade variou de 5 a 98 anos, com média de $60,27$ (desvio padrão de $\pm 16,79)$, e 39 (10,99\%) tinham até 40 anos. Destes, $19(48,72 \%)$ eram mulheres e 20 $(51,28 \%)$ eram homens.

A maioria dos CCRs foi diagnosticada entre 40 e 80 anos (figura 2) e, em $178(50,14 \%)$ casos a procedência foi de Aracaju.

Adenocarcinoma foi, significativamente, o tipo histológico mais encontrado, presente em $295(83,10 \%)$ pacientes, seguido de tumor carcinóide $(\mathrm{n}=16 ; 4,51 \%)$ e carcinoma de células escamosas $(n=14 ; 3,94 \%)$ (fi-

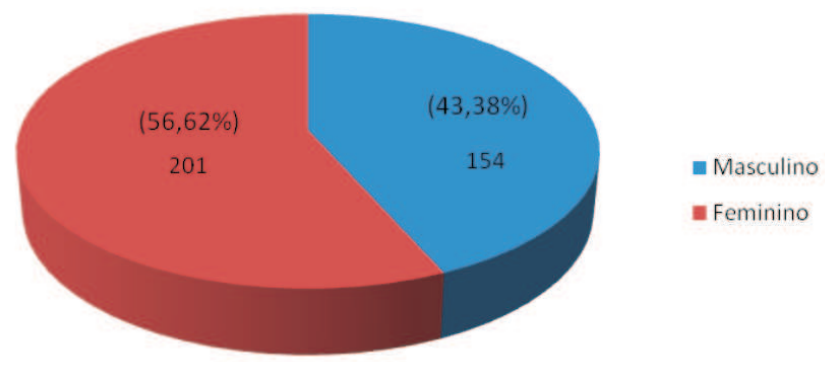

Figura 1 - Incidência de câncer colorretal por gênero no período de 2003 a 2005 em Sergipe. 
gura 3). Dos que apresentaram adenocarcinoma, os moderadamente diferenciados $(n=146 ; 49,49 \%)$ foram o grau de diferenciação mais comuns, seguido dos bem diferenciados $(\mathrm{n}=81 ; 27,46 \%)$ e pouco diferenciados $(\mathrm{n}=17 ; 5,76 \%)$. Em 51 (17,29\%) casos não havia informação a respeito do grau de diferenciação (figura 4).

Com relação à localização do tumor, dentre aqueles que apresentaram adenocarcinoma, houve um predomínio do reto $(\mathrm{n}=121 ; 41,02 \%)$, seguido do sigmóide $(n=38 ; 12,88 \%)$ e ceco $(n=30 ; 10,17 \%)$ (figura 5). Em 31 casos (10,51\%) não foi especificado. Agrupando os tumores em cólons direito e esquerdo, notou-se prevalência deste último com 187 (63,39\%) casos diagnosticados, contra $88(29,83 \%)$ casos do cólon direito. Em $20(6,78 \%)$ casos não foi especificado o sítio do tumor (figura 6).

Foi observado um aumento nos tumores do cólon direito em relação ao esquerdo no período estudado. Em 2003, 2004 e 2005, excluindo-se os casos em que não havia especificado o local do intestino grosso

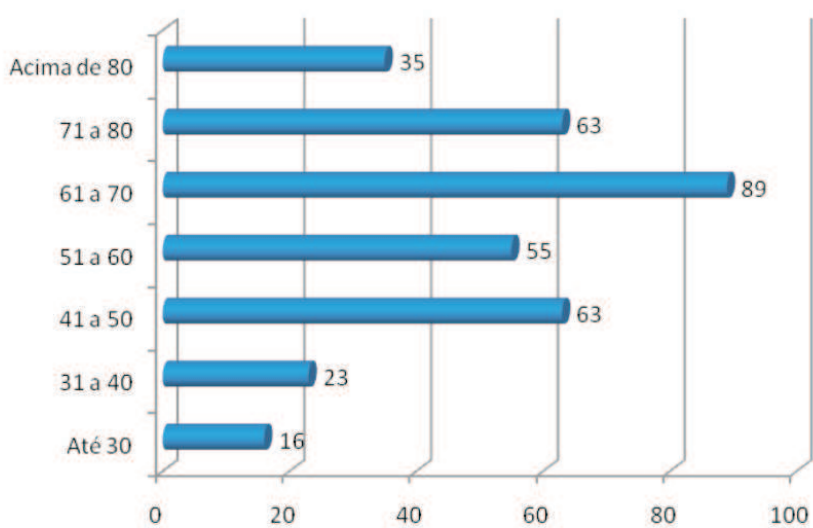

Figura 2 - Distribuição de câncer colorretal segundo a idade dos pacientes, estratificada por décadas.

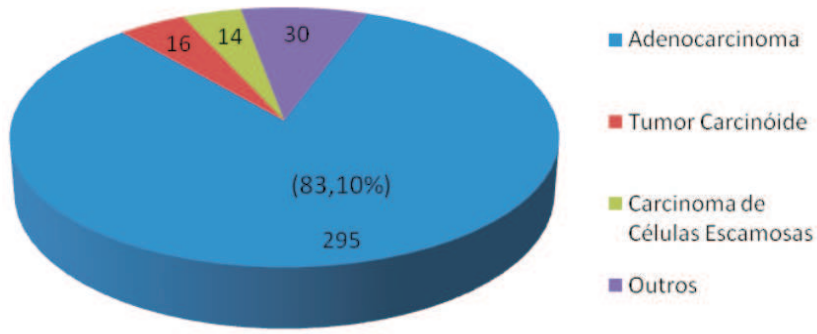

Figura 3 - Distribuição do câncer colorretal quanto ao tipo histológico. acometido ( $\mathrm{n}=22 ; 6,20 \%)$, o cólon direito foi o sítio do tumor em 32,32; 34,62 e 34,62\% dos casos, respectivamente. Já o cólon esquerdo foi acometido em 67,68; 65,38 e $65,38 \%$ no respectivo período estudado (tabela 1 e figura 7).

Em 184 (51,83\%) casos havia informação do estado civil e, em 155 (43,66\%), da raça. Dos casos que havia informação a respeito do estado civil e raça, observou-se que $127(69,02 \%)$ eram casados, 31 $(16,85 \%)$ eram solteiros, $20(10,87 \%)$ eram viúvos e 6 $(3,26 \%)$ eram divorciados. Quanto à raça, $83(53,55 \%)$ eram brancos, $70(45,16 \%)$ eram pardos e $2(1,29 \%)$ negros.

Em $134(37,75 \%)$ casos havia informação a respeito do estádio do tumor no momento do diagnóstico. Houve uma predominância de tumores em estádio III $(\mathrm{n}=61 ; 45,52 \%)$, seguindo-se o estádio II $(\mathrm{n}=49$; $36,57 \%)$, estádio I $(n=17 ; 12,69 \%)$, estádio IV $(n=6$; $4,48 \%)$ e estádio $0(n=1 ; 0,75 \%)$ (figura 8). Quanto à profundidade do tumor segundo o sistema TNM, foi

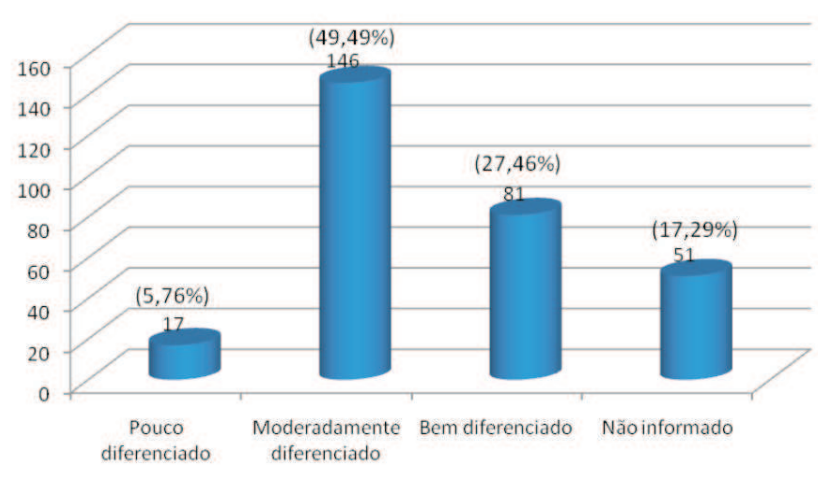

Figura 4 - Distribuição de adenocarcinoma quanto ao grau de diferenciação.

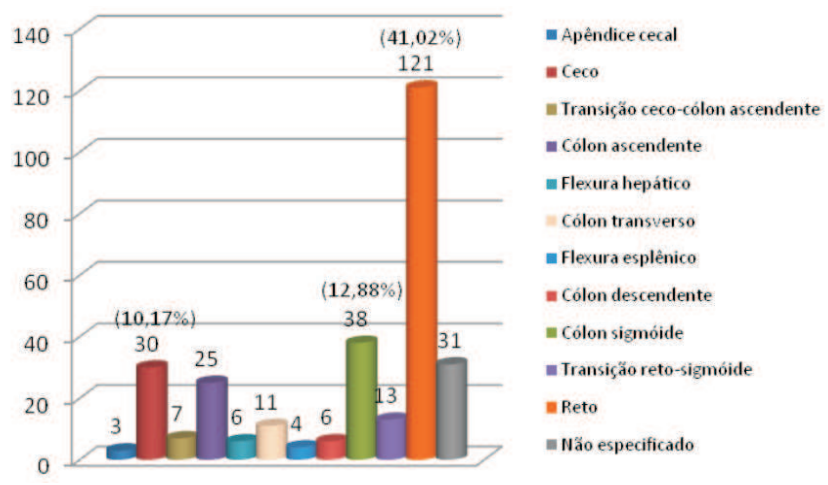

Figura 5 - Distribuição de adenocarcinoma quanto à localização. 


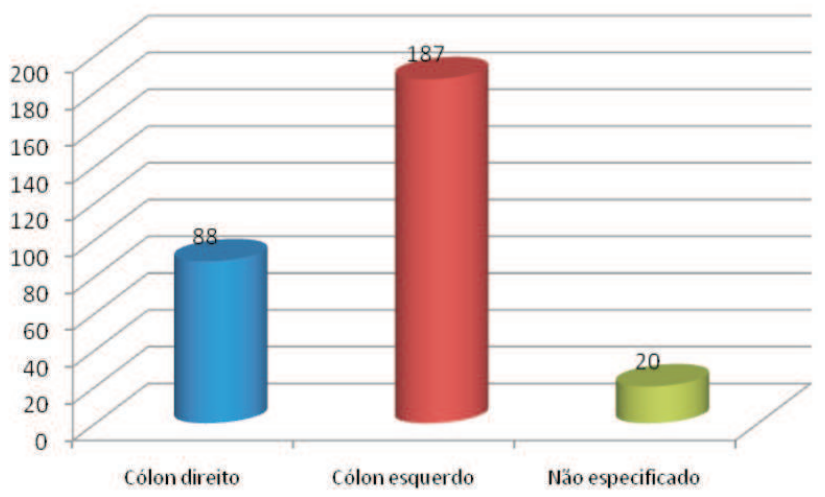

Figura 6-Distribuição de adenocarcinoma quanto à localização.

possível identificar $94(26,48 \%)$ casos. A maioria dos casos foi classificada como pT3 (n=69; 73,40\%), seguido de pT2 (n=17; 18,09\%), pT4 (n=4; 4,26\%), pT1 $(\mathrm{n}=3 ; 3,19 \%)$ e pTis $(\mathrm{n}=1 ; 1,06 \%)$ (figura 9). Considerando apenas os que apresentam adenocarcinoma, em 88 casos $(24,79 \%)$ tiveram informação a respeito do estadiamento de Dukes. Houve um predomínio de Dukes C ( $\mathrm{n}=46 ; 52,27 \%)$ e B ( $\mathrm{n}=34 ; 38,64 \%)$, seguido de Dukes A ( $n=4 ; 4,55 \%)$ e D ( $n=4 ; 4,55 \%)$.

No estado de Sergipe, a incidência de CCR em 2003, 2004 e 2005 foi de 4,57; 4,72 e 5,29 por 100.000 homens e, 6,07; 5,57 e 6,98 por 100.000 mulheres respectivamente (figura 10). Na capital, nos respectivos anos, esses números aumentaram para 10,25; 10,57 e 10,29 por 100.000 homens e 12,$92 ; 13,16$ e 15,07 por 100.000 mulheres (figura 11). Independentemente do gênero, a incidência da patologia no estado em 2003, 2004, 2005 foi de 10,$64 ; 10,28$ e 12,27 por 100.000 pessoas, respectivamente, tendo incidência média de 11,06 (desvio padrão de $\pm 1,05$ ). Na capital, nos respectivos anos, a incidência foi de 23,17; 23,72 e 25,36 por 100.000 pessoas, e média de 24,09 (desvio padrão de $\pm 1,14$ ). Analisando apenas a incidência do grupo etário maior ou igual a 50 anos, o risco de desenvolver CCR nesta faixa etária específica em 2003, 2004 e 2005 foi de 30,$00 ; 29,59$ e 37,01 por 100.000 pessoas no estado e, 57,$24 ; 55,08$ e 68,13 por 100.000 pessoas na capital, respectivamente (figura 12 ).

\section{DISCUSSÃO}

O estudo analisou 355 pacientes, sendo 201 $(56,62 \%)$ mulheres e $154(43,38 \%)$ homens, perfil concordante com dados disponibilizados pelo INCA ${ }^{(5)}$. A

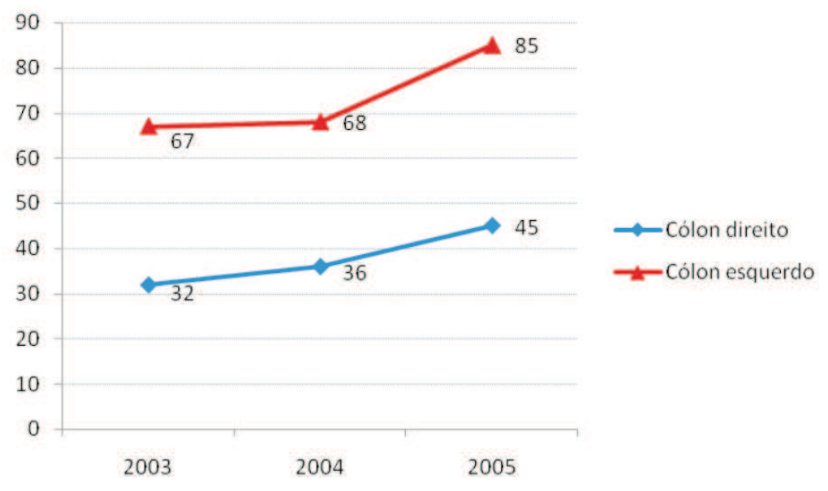

Figura 7 - Incidência de câncer colorretal nos cólons direito e esquerdo no período estudado (2003 a 2005).

Tabela 1 - Distribuição do câncer colorretal nos cólons direto e esquerdo no período estudado (2003 a 2005).

\begin{tabular}{lcc}
\hline Ano & & $\mathbf{2 0 0 3}$ \\
\hline Cólon direito & 32 & $32,32 \%$ \\
Cólon esquerdo & 67 & $67,68 \%$ \\
Total & 99 & $100 \%$ \\
\hline Ano & & $\mathbf{2 0 0 4}$ \\
\hline Cólon direito & 36 & $34,62 \%$ \\
Cólon esquerdo & 68 & $65,38 \%$ \\
Total & 104 & $100 \%$ \\
\hline Ano & & $\mathbf{2 0 0 5}$ \\
\hline Cólon direito & 45 & $34,62 \%$ \\
Cólon esquerdo & 85 & $65,38 \%$ \\
Total & 130 & $100 \%$ \\
\hline
\end{tabular}

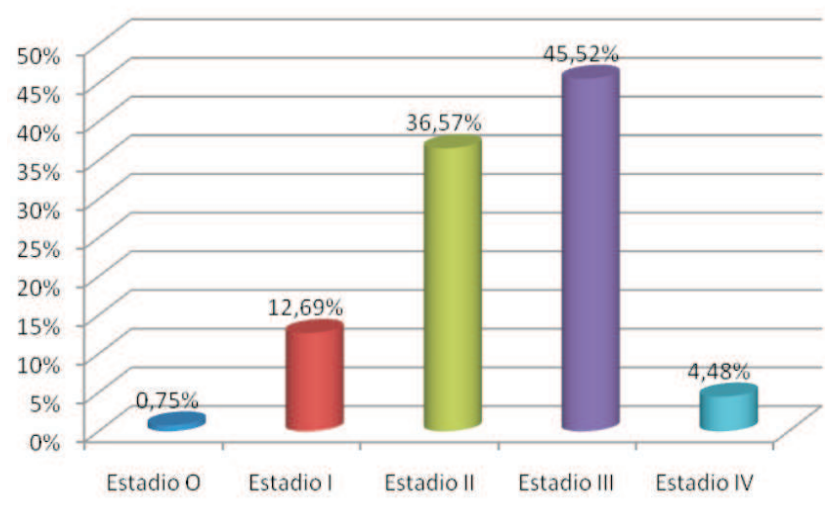

Figura 8 - Distribuição do câncer colorretal em relação ao estádio do tumor no momento do diagnóstico. 


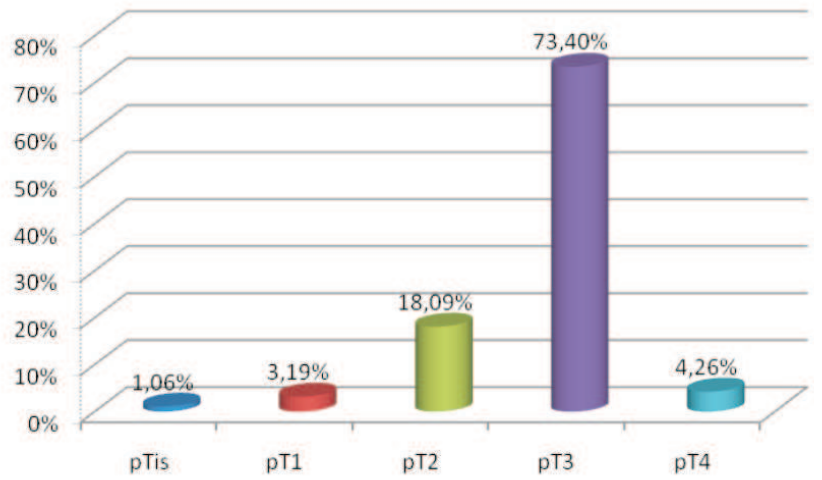

Figura 9-Distribuição do câncer colorretal em relação à profundidade do tumor ( $p T$ ) segundo o TNM.

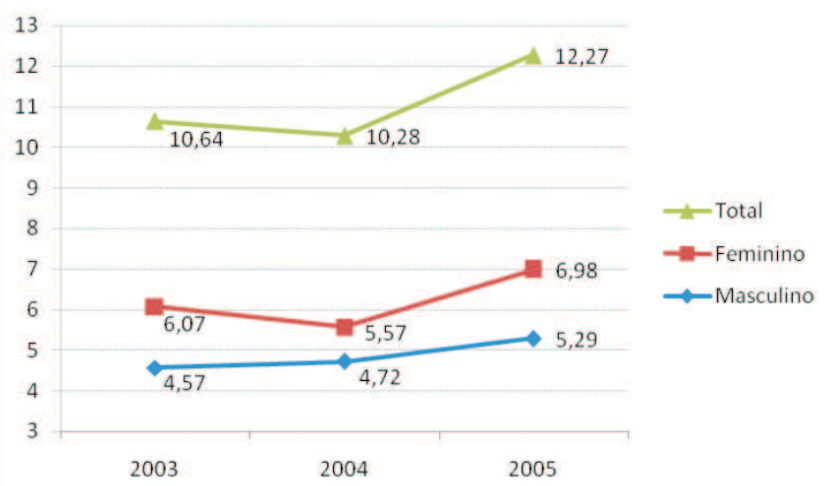

Figura 10 - Incidência de câncer colorretal por 100.000 habitantes em relação ao gênero no estado de Sergipe.

estatística americana também mostra um padrão semelhante, observado por Devessa et al ${ }^{(8)}$, o que difere da estatística japonesa, onde há uma prevalência do gênero masculino em relação ao gênero feminino, observado por Tamura et al ${ }^{(9)}$.

A população mais acometida tinha entre $40 \mathrm{e}$ 80 anos, com pico de incidência na sétima década de vida, sendo a média de idade de 60,27 anos, semelhante à encontrada na literatura ${ }^{(3,4,10,11,12)}$. Dados da Sociedade Americana de Câncer apresenta uma média de idade ao diagnóstico de 71 anos. Em nossa casuística, 39 pacientes tinham até 40 anos, o que representa $10,99 \%$ da amostra, sendo $19(48,72 \%)$ mulheres e 20 $(51,28 \%)$ homens. Mauad et al. mostraram incidência semelhante, de $12,6 \%$, de pacientes com até 40 anos, com discreta predominância do gênero masculino $(51,22 \%)^{(13)}$.

Com relação à procedência, houve uma predominância de pacientes provenientes da capital $(50,14 \%)$, sendo que em $16(4,51 \%)$ casos não foi possível obter esta informação.

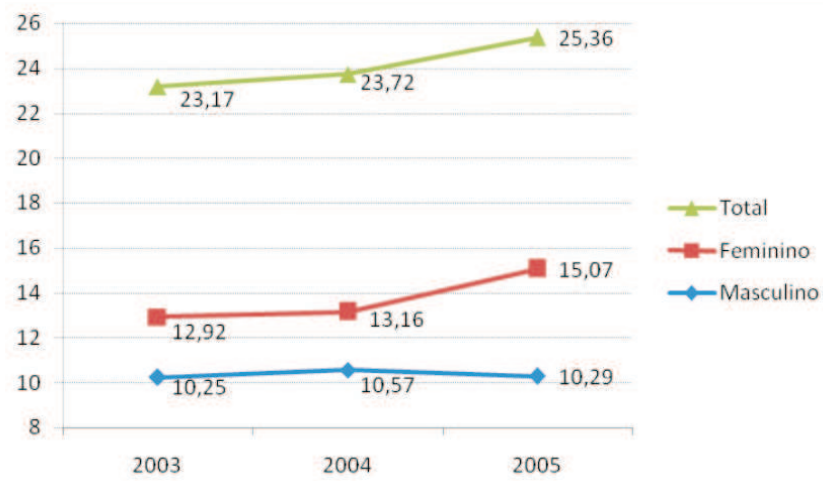

Figura 11 - Incidência de câncer colorretal por 100.000 habitantes em relação ao gênero na capital.

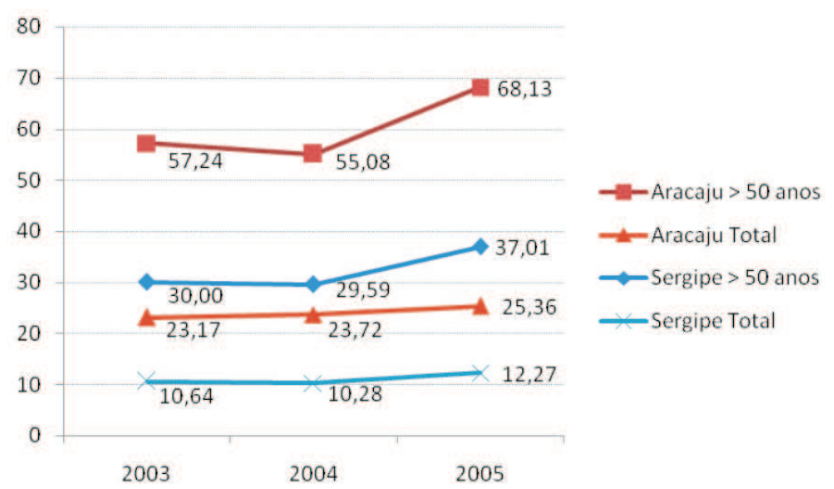

Figura 12 - Comparativo da incidência total e específica para pacientes acima de 50 anos em Sergipe e Aracaju.

Adenocarcinoma foi significativamente o tipo histológico mais encontrado, presente em 295 (83,10\%) pacientes, padrão semelhante à encontrada na literatu$\mathrm{ra}^{(3)}$. Dos que apresentaram adenocarcinoma, os moderadamente diferenciados $(n=146 ; 49,49 \%)$, foram o grau de diferenciação mais comum, seguido dos bem diferenciados $(\mathrm{n}=81 ; 27,46 \%)$ e pouco diferenciados $(\mathrm{n}=17 ; 5,76 \%)$. Em $51(17,29 \%)$ casos não havia informação a respeito do grau de diferenciação. Júnior et al. mostraram uma gradação na qual os moderadamente diferenciados $(69,8 \%)$ foram mais freqüentes, seguido dos bem diferenciados $(22,6 \%)$ e dos pouco diferenciados ou indiferenciados $(5,1 \%)^{(3)}$.

Quanto à distribuição topográfica dos adenocarcinomas, o reto foi o sítio mais comum, presente em $121(41,02 \%)$ dos pacientes, seguido do sigmóide $(\mathrm{n}=38 ; 12,88 \%)$ e ceco $(\mathrm{n}=30 ; 10,17 \%)$. Notou-se, então, um predomínio de tumores do cólon esquerdo $(\mathrm{n}=187 ; 63,39 \%)$, contra o cólon direito $(\mathrm{n}=88$; $29,83 \%$ ). Esses dados são semelhantes à literatura brasileira ${ }^{(3,4,10)}$, onde o reto é o local mais acometido. Dados 
americanos apontam para uma maior frequiência de tumores em sigmóide, seguido do reto e ceco ${ }^{(8,14)}$. A literatura tem apontado para um aumento da incidência de tumores no cólon direito nos últimos anos ${ }^{(15)}$, fato observado em nossa casuística, quando foi comparada a incidência de tumores do cólon esquerdo com o direito no período de 2003 a 2005.

Em nosso estudo, a maioria dos tumores foi detectada em fases mais avançadas da doença. Houve uma predominância de tumores em estádio III ( $\mathrm{n}=61$; $45,52 \%)$, seguindo-se o estádio II ( $\mathrm{n}=49 ; 36,57 \%)$, estádio I ( $\mathrm{n}=17 ; 12,69 \%)$, estádio IV $(\mathrm{n}=6 ; 4,48 \%)$ e estádio $0(\mathrm{n}=1 ; 0,75 \%)$. Estádios mais avançados no momento do diagnóstico também foram observados em outros estudos, o que reflete diretamente em um pior prognóstico da doença. A Sociedade Americana de Câncer observou que 39\% dos casos de CCR foram diagnosticados quando o câncer ainda estava confinado ao sítio primário (estádio localizado), $36 \%$ foram diagnosticados depois que o câncer espalhou para os linfonodos regionais ou diretamente além do sítio primário e 19\% foram diagnosticados depois que o câncer já tinha metastatizado (estadio avançado). As taxas relativas correspondentes de uma sobrevivência de 5 anos foram de $89,8 \%$ para localizado, $67,7 \%$ para regional, $10,3 \%$ para distante e $35,8 \%$ para desconhecidos ${ }^{(16)}$. Ressalta-se aqui a importância das políticas de saúde na detecção e tratamento precoce do CCR, melhorando o prognóstico e sobrevida desses pacientes. Já existe um trabalho sendo desenvolvido em parceria com a Sociedade Brasileira de Coloproctologia na prevenção do câncer colorretal. Apesar de relatarmos neste estudo uma incidência elevada de câncer em estádio avançado, temos acompanhado, através do serviço de colonoscopia dos diversos centros do nosso estado, o diagnóstico e tratamento endoscópico de adenomas e adenocarcinomas, que com certeza já es- tão interferindo de forma positiva no controle da doença, embora ela ainda assim esteja aumentando a sua incidência.

Quanto à profundidade do tumor segundo o sistema TNM, a maioria dos casos foi classificada como pT3 $(n=69 ; 73,40 \%)$, seguido de pT2 $(n=17 ; 18,09 \%)$, pT4 $(n=4 ; 4,26 \%)$, pT1 $(n=3 ; 3,19 \%)$ e pTis $(n=1$; $1,06 \%$ ). Dos que apresentaram adenocarcinoma, houve um predomínio de Dukes C $(\mathrm{n}=46 ; 52,27 \%)$ e B $(\mathrm{n}=34 ; 38,64 \%)$, seguido de Dukes A $(\mathrm{n}=4 ; 4,55 \%)$ e D $(\mathrm{n}=4 ; 4,55 \%)$. Júnior et al também mostraram comportamento semelhante, sendo pT3 responsável por $64,7 \%$ do total e, Dukes B, C e D, por $85 \%$ dos casos, o que correspondeu a um prognóstico mais reservado ${ }^{(3)}$.

Foi possível calcular a incidência anual, pois os dados foram colhidos a partir dos laudos de todos os laboratórios de anatomia patológica do estado. Dados do INCA estimaram no estado para o ano 2003 que a taxa bruta de incidência por 100.000 habitantes fosse de 2,12 para homens e 3,20 para mulheres. Na capital, esses números aumentaram para 4,70 para homens e 8,51 para mulheres. O que corresponde a 20 casos do gênero masculino no estado e 10 na capital contra 30 casos do gênero feminino no estado e 20 na capital ${ }^{(17)}$. Nosso estudo revelou uma incidência maior tanto no estado quanto na capital. A incidência de CCR no estado para uma população de 100.000 foi de 4,57 e 6,07 para homens e mulheres, respectivamente, em 2003. Na capital, esses números aumentaram para 10,25 para homens e 12,92 para mulheres (tabela 2). O que correspondeu a 42 casos do gênero masculino e 23 na capital contra 58 casos do gênero feminino no estado e 33 na capital. Em 2004 e 2005, a incidência de CCR no estado foi 4,72 e 5,29 por 100.000 homens e, 5,57 e 6,98 por 100.000 mulheres, respectivamente. Na capital, nos respectivos anos, esses números aumentaram para 10,57 e 10,29 por 100.000 homens e 13,16 e 15,07

Tabela 2 - Comparativo das incidências encontradas com as estimadas pelo INCA no período de 2003 em Sergipe e Aracaju.

\begin{tabular}{llll}
\hline INCA & \multicolumn{2}{c}{ Estudo Demográfico } \\
\hline Sergipe & & & \\
Homens & $2,12 / 100.000$ & Homens & $4,57 / 100.000$ \\
Mulheres & $3,20 / 100.000$ & Mulheres & $6,07 / 100.000$ \\
Aracaju & & & \\
Homens & $4,70 / 100.000$ & Homens & $10,25 / 100.000$ \\
Mulheres & $8,51 / 100.000$ & Mulheres & $12,92 / 100.000$ \\
\hline
\end{tabular}


por 100.000 mulheres. As incidências observadas no estado foram bem menores do que as encontradas nas estatísticas americanas ${ }^{(16)}$ e japonesa ${ }^{(9)}$. No entanto, esses valores foram bem maiores que as estimadas pelo INCA para a região nordeste ${ }^{(5)}$.

Como a maioria dos protocolos de rastreamento do CCR preconiza pesquisar pacientes assintomáticos e sem fatores de risco, acima dos 50 anos de idade ${ }^{(18)}$, analisamos esse grupo etário em separado. A análise da incidência desse grupo foi calculada através da divisão do número de casos novos com idade igual ou superior a 50 anos pela população residente referente a este grupo etário no estado e capital nos anos de 2003, 2004 e 2005. Foi observado o risco de desenvolver CCR de 30,00; 29,59 e 37,01 por 100.000 pessoas no estado e, 57,24; 55,08 e 68,13 por 100.000 pessoas na capital, respectivamente.

No serviço de coloproctologia do Hospital Universitário da UFS, criamos um ambulatório de referência para acompanhamento dos pacientes portadores de neoplasia do cólon, reto e ânus com o objetivo de otimizar políticas de prevenção e tratamento desses pacientes. A idéia é priorizar o atendimento inicial de todo paciente encaminhado ao serviço com suspeita de neoplasia, e posterior padronização do seu atendimento.

Buscaremos manter atualizado nosso banco de dados, para que em futuras publicações ou reuniões sobre o tema tenhamos uma estatística e dados demográficos mais próximos da nossa realidade que dados de outros países.

\section{CONCLUSÕES}

Foram encontrados 355 casos de câncer de cólon e reto, havendo uma maior prevalência em pacientes de gênero feminino $(56,62 \%)$ em relação ao gênero masculino $(43,38 \%)$. O pico de incidência ocorreu na sétima década de vida, sendo a média de idade ao diagnóstico de 60,27 anos. Adenocarcinoma foi significativamente o tipo histológico mais encontrado, presente em $295(83,10 \%)$ pacientes. Destes, o reto foi o local mais acometido, presente em $121(41,02 \%)$ pacientes. Foi observado um aumento da incidência de tumores no cólon direito. A maioria dos tumores foi detectada em fases mais avançadas da doença, havendo uma predominância de tumores em estádio III $(45,52 \%)$. Houve um aumento nas incidências anuais da neoplasia tanto no estado quanto na capital. Nossos dados revelaram uma incidência maior que as estimadas pelo INCA tanto em Sergipe quanto em Aracaju. No estado de Sergipe, a incidência de CCR em 2003, 2004 e 2005 foi de 4,$57 ; 4,72$ e 5,29 por 100.000 homens e, 6,07; 5,57 e 6,98 por 100.000 mulheres respectivamente. Na capital, nos respectivos anos, esses números aumentaram para 10,$25 ; 10,57$ e 10,29 por 100.000 homens e 12,92 ; 13,16 e 15,07 por 100.000 mulheres.

\footnotetext{
ABSTRACT: In Brazil, the colorretal cancer (CRC) was the fifth cause of death for cancer in men and fourth, in women in the period of 1995-1999. However, there are few registers on the epidemiologicals aspects of this pathology in Sergipe, being this the focus of this study. Objective: To catalogue all CRC cases diagnosed in Sergipe in the period of 03 years (2003-2005), tracing a demographic study. Method: The data had been harvested in findings of all pathological anatomy laboratories on the state. Results: The CRC was more frequent in women $(n=201 ; 56,62 \%)$ than in men $(n=154 ; 43,38 \%)$. The average age was 60,27 years old, and $39(10,99 \%)$ had up to 40 years old. Adenocarcinoma was the histology type more found, present in $295(83,10 \%)$ patients. In Sergipe, the incidence of $C R C$ in 2003, 2004 and 2005 was 4,57; 4,72 and 5,29 per 100.000 men and, 6,07; 5,57 and 6,98 per 100.000 women respectively. In the capital, in this respectives years, these numbers had increased to 10,$25 ; 10,57$ and 10,29 per 100.000 men and 12,92; 13,16 and 15,07 per 100.000 women. Conclusions: The achieved data are similar to the ones of the literature, showing an increase in the annual incidences of the neoplasm.
}

Key words: Cancer, colon, rectum, epidemiology.

\section{REFERÊNCIAS}

1. Brasil. Ministério da Saúde. Sistema de Informações sobre Mortalidade. Disponível em: <http://portal.saude.gov.br/portal/arquivos/pdf/mortalidade\%202001.pdf $>$. Acesso em: 26 nov. 2006.
2. Brasil. Ministério da Saúde. Instituto Nacional do Câncer. Distribuição proporcional do total de mortes por câncer, segundo localização primária do tumor, em homens e mulheres, para os períodos entre 1979 e 1983 e entre 1995 e 1999. Disponível em: <http://www.inca.gov.br/atlas/docs/ distrib_prop_BR.pdf $>$. Acesso em: 26 nov. 2006. 
3. Júnior TP, Alves AJC, Nogueira AMMF. Câncer colorretal: análise anatomopatológica de 476 colectomias consecutivas em Belo Horizonte (MG). J Bras Patol Med Lab 2005; 41(3): p 175-184.

4. Pinho MSL, Ferreira LC, Brigo MJK, Pereira Filho A, Wengerkievicz A, Ponath A, et al. Incidência do câncer colorretal na regional de saúde de Joinville (SC). Rev bras Coloproct 2003; 23(2): p 73-76.

5. Brasil. Ministério da Saúde. Instituto Nacional do Câncer. Estimativa 2006 - Estimativa da Incidência de Câncer no Brasil. Disponível em: <http://www.inca.gov.br/estimativa/ 2006/versaofinal.pdf> . Acesso em: 03 nov. 2006.

6. Neves FJ, Mattos IE, Koifman RJ. Mortalidade por câncer de cólon e reto nas capitais brasileiras no período 1980-1997. Arq Gastroenterol 2005; 42(1): p 63-70.

7. População Residente em Sergipe. Censos Demográficos e Contagem Populacional; para os anos intercensitários, estimativas preliminares dos totais populacionais, estratificados por idade e sexo pelo MS/SE/Datasus. Disponível em: <http:// tabnet.datasus.gov.br/cgi/tabcgi.exe?ibge/cnv/popse.htm>. Acesso em: 24 jun. 2007.

8. Devessa SS, Chow W. Variation in colorectal cancer incidence in the United States by subsite of origin. Cancer 1993; 71(12): p 3819-3826.

9. Tamura K, Ishiguro S, Munakata A, Yoshida Y, Nakaji S, Sugawara K. Annual changes in colorectal carcinoma incidence in Japan. Cancer 1996; 78(6): p 1187-1194.

10. Cruz GMG. Propedêutica colorretal. In: Coloproctologia Propedêutica Nosológica. 2000. p 835-921.

11. Raso P, Nogueira AMMF, Filho GB, Barbosa AJA, editors. Tumores Malignos. In: Bogliolo Patologia. $6^{\mathrm{a}}$ ed. 2000. p 632-25.
12. Dubois, RN. In: Goldman L, Ausiello D, editors. Adenocarcinoma de cólon e reto. Cecil Tratado de Medicina. $22^{\mathrm{a}}$ ed. 2005. p 1406-10.

13. Mauad EC, Gomes UA, Boldrini D, Haikel R, Santiago GH, Maruta LM. Câncer de cólon e reto em pacientes abaixo dos 40 anos. Revista Brasileira de Cancerologia 2000; 46(4): p 371-376.

14. Demers RY, Severson RK, Schottenfeld D, Lazar L. Incidence of colorectal adenocarcinoma by anatomic subsite. Cancer 1997; 79(3): p 441-447.

15. Ponz de Leon M, Marino M, Benatti P, Rossi G, Menigatti M, Pedroni M, et al. Trend of incidence, subsite distribution and staging of colorectal neoplams in the 15-year experience of a specialized cancer registry. Ann Oncol 2004; 15(16): p 940-946.

16. U.S. National Institutes of Health. National Cancer Institute. Cancer of the Colon and Rectum. Disponível em: <http://seer.cancer.gov/statfacts/html/ colorect.html?statfacts_page $=$ colorect.html $\& x=13 \& y=15>$. Acesso em: 29 abr. 2007.

17. Brasil. Ministério da Saúde. Instituto Nacional do Câncer. Estimativa 2003 - Estimativa da Incidência de Câncer no Brasil. Disponível em: <http://www.inca.gov.br/estimativas/ 2003/index.asp?link=tabelaestados.asp\&UF=SE > . Acesso em: 19 de ago. 2007.

18. Mayer RJ. Câncer Colorretal. In: Harrison Medicina Interna. $16^{a}$ ed. 2006. p 553- 558.

\section{Endereço para Correspondência:}

FÁBIO RAMOS TEIXEIRA

Av. Oceânica, 1077, Apt. 401, Bloco A, Bairro: Atalaia Velha 49035-000 Aracaju-SE

Ramos15@ superig.com.br 